\title{
La protection du migrant « non-éloignable » en raison de son état de santé dans la jurisprudence européenne
}

The Non-Expulsive Migrant Protection because of his State of Health in European Case Law

La protección del migrante no expulsivo debido a su estado de salud en la jurisprudencia europea

\section{Cassandre Genonceau}

\section{(2) OpenEdition \\ Journals}

Édition électronique

URL : https://journals.openedition.org/remi/11013

DOI : $10.4000 /$ remi. 11013

ISSN : $1777-5418$

Éditeur

Université de Poitiers

Édition imprimée

Date de publication : 1 octobre 2018

Pagination : 319-333

ISBN : 979-10-90426-62-7

ISSN : 0765-0752

Référence électronique

Cassandre Genonceau, «La protection du migrant « non-éloignable » en raison de son état de santé dans la jurisprudence européenne », Revue européenne des migrations internationales [En ligne], vol. 34 $n^{\circ} 2$ et $3 \mid 2018$, mis en ligne le 01 décembre 2018, consulté le 14 avril 2022. URL : http:// journals.openedition.org/remi/11013; DOI : https://doi.org/10.4000/remi.11013 


\section{Chronique juridique}

\section{La protection du migrant « non- éloignable " en raison de son état de santé dans la jurisprudence européenne}

\section{Cassandre Genonceau ${ }^{1}$}

Les principaux traités internationaux et régionaux relatifs aux droits de I'Homme consacrent un droit à la protection de la santé - y compris mentale - et à I'assistance médicale ${ }^{2}$. Ils s'appliquent aux migrants ${ }^{3}$ indépendamment de leur nationalité ou de leur situation administrative, dès lors qu'ils relèvent effectivement de la juridiction des États parties (lorsqu'ils sont placés sous leur contrôle exclusif et continu pendant la période considérée). Toutefois, tous les États sont en réalité libres d'apprécier la manière dont ce droit doit être appliqué. Ces textes ne consacrent aucun niveau minimal de traitement, et l'accès aux soins primaires et d'urgence aux étrangers en situation irrégulière peut être soumis à des restrictions jugées proportionnées et raisonnables.

Au niveau du Conseil de l'Europe, la Convention de sauvegarde des droits de I'Homme et des libertés fondamentales («la Convention») ne consacre pas de droit à la protection de la santé. Toutefois, certaines dispositions sont susceptibles d'être liées à la santé physique et mentale. C'est le cas de l'article 2 consacré au droit à la vie, de l'article 3 consacré à l'interdiction des traitements inhumains ou dégradants ou encore de l'article 8 relatif au droit au respect de la vie privée et familiale.

Au niveau de I'Union européenne ( "I'UE »), la Directive Retour ${ }^{4}$, régissant le retour forcé des ressortissants de pays tiers à I'Union en situation irrégulière sur le territoire de ses États membres, permet à ces derniers de reporter l'éloignement du migrant pour des "motifs justifiés de non-retour», notamment fondés

\footnotetext{
1 Doctorante en droit privé, Laboratoire AMURE (Brest), Attachée temporaire d'enseignement et de recherche en droit privé (Sorbonne, Paris I) Faculté de droit, Université du Caire, Giza, Le Caire, Égypte ; cassandre.genonceau@gmail.com

2 Voir notamment PIDESC (1966), art. 12; Convention sur la protection des droits de tous les travailleurs migrants et des membres de leur famille (1990), art. 28; Charte des droits fondamentaux de l'Union européenne (2000), art. 35.

3 Par souci de simplification, le terme "migrant " sera employé au sens large. Consacré dans certains glossaires et textes déclaratoires d'organisations internationales (UNESCO; OIM, etc.), ce terme désigne les individus traversant des frontières internationales pour s'établir dans un pays étranger. Toutefois, il est nécessaire de souligner dès à présent qu'il ne correspond pas stricto sensu à la catégorie juridique qui doit être retenue une fois qu'il a achevé son déplacement («immigrant», en situation régulière ou non).

4 Directive 2008/115/CE («Directive Retour»).
} 
sur des circonstances humanitaires personnelles à l'individu tel que son état de santé physique ou mental.

À défaut de la suspension de son renvoi par les autorités nationales, le migrant malade peut former une requête exceptionnelle en mesures provisoires et/ou une requête individuelle visant à engager la responsabilité d'un État partie à la Convention devant la Cour Européenne des Droits de l'Homme (CEDH). Pour prévenir ou réparer la violation d'un droit fondamental consacré dans la Convention, les décisions de la CEDH peuvent conduire à la suspension provisoire et en urgence de l'éloignement d'un migrant en situation irrégulière gravement malade sur le territoire d'un État partie. Dans certaines circonstances, la Cour de Justice de I'Union Européenne (CJUE) impose également aux États membres de l'Union européenne de suspendre l'éloignement des migrants en situation irrégulière sur leur territoire pour des motifs médicaux ${ }^{5}$.

\section{L'éloignement du migrant malade sous l'angle de l'interdiction des traitements inhumains et/ou dégradants (article 3 de la Convention et article 4 de la Charte)}

En principe, un migrant malade en situation irrégulière ne peut revendiquer un droit au maintien sur le territoire d'accueil dans le seul but de bénéficier de la sécurité sociale, des soins médicaux ou de la protection médicale de ce pays, dont il ne pourrait pas jouir dans son pays d'origine. II reste que dans certaines hypothèses, l'éloignement d'un migrant malade peut être assimilé à un traitement inhumain ou dégradant si le retour forcé se révèle dangereux pour sa dignité ou sa vie, au sens de la Convention (article 3) ou de la Charte (article 4) qui prohibent un tel traitement.

Si la CEDH et la CJUE ont progressivement assoupli leurs positions, la suspension de la mesure d'éloignement, d'expulsion ou d'extradition de migrants malades, ou la responsabilité de l'État partie pour l'avoir mise en œuvre, demeure encore très exceptionnelle.

\section{L'hypothèse très exceptionnelle de suspension de l'éloignement d'un migrant malade}

Cette interdiction des traitements inhumains ou dégradants est absolue, c'est-à-dire qu'elle ne peut souffrir d'aucune dérogation ou assouplissement. Les juridictions européennes considèrent toutefois que le traitement litigieux doit revêtir une gravité suffisante pour constituer un traitement inhumain ou dégradant.

Conformément à son modus operandi, la CEDH procède à une appréciation in concreto des faits soumis à son examen. Dès lors, chaque situation peut

5 La CEDH peut être saisie directement par tout individu, quelle que soit sa nationalité, dès lors qu'il relève de la juridiction d'un État partie à la Convention dont il estimerait avoir subi un préjudice. En revanche, aucun recours individuel direct ne peut être formé par une personne physique devant la CJUE. Les jurisprudences pertinentes relatives aux droits des migrants sont généralement, celles issues des questions préjudicielles posées à la CJUE par les juges nationaux des Etats membres avant de rendre leur décision. 
donner lieu à des décisions parfois très différentes. Le degré de gravité du mauvais traitement allégué doit être apprécié comme suffisamment grave par la CEDH pour emporter violation de l'article 3 si l'éloignement devait être rendu effectif : tout dépendra des faits et de l'impact de l'éloignement sur l'individu. Par ailleurs, ce qui pourra être considéré par l'individu comme subjectivement humiliant (caractère dégradant) ou portant atteinte à son intégrité physique ou psychologique (caractère inhumain) ne le sera pas forcément perçu comme tel objectivement par la CEDH. En somme, il convient de distinguer la nature de l'obligation, qui est absolue, de l'appréciation des conséquences susceptibles de découler si celle-ci était violée, qui s'avère relative.

En principe, la CEDH apprécie l'état de santé de la victime, sa situation familiale et individuelle, la nature et le contexte du traitement, et ses effets physiques ou mentaux. S'agissant plus particulièrement du migrant malade, la CEDH a défini puis précisé ce seuil de gravité dans quatre affaires importantes, dont trois impliquant le Royaume-Uni : D. contre Royaume-Uni (1997), Bensaid contre Royaume-Uni (2001), N. contre Royaume-Uni (2008) et S. J. contre Belgique (2014).

La première affaire concernait la décision des autorités britanniques d'expulser M. D vers Saint-Kitts. Atteint d'une maladie incurable au stade terminal $(\mathrm{VIH})$, il alléguait du risque réel d'être soumis aux mauvais traitements visés par l'article 3, dès lors qu'il n'était pas certain de bénéficier là-bas de soins médicaux ou infirmiers d'une part, et d'un soutien familial, amical ou social désireux ou en mesure de s'occuper de lui d'autre part. Pour caractériser un traitement inhumain, la juridiction s'est alors fondée sur le risque réel pour $M$. D de mourir dans des conditions particulièrement douloureuses s'il était expulsé, au moment où elle apprécie les faits. Elle releva que I'affaire était marquée par des "circonstances très exceptionnelles", dans lesquelles pouvaient entrer en jeu des "considérations humanitaires impérieuses" militant contre son expulsion, sans toutefois définir ces notions.

La deuxième affaire concernait I'expulsion imminente par le Royaume-Uni de $M$. Bensaid, étranger en situation irrégulière schizophrène, vers l'Algérie. M. Bensaid alléguait que s'il y était renvoyé, il ne disposerait pas des soins adéquats en l'absence de voiture personnelle lui permettant de se rendre à I'hôpital le plus proche en mesure de dispenser un traitement psychiatrique (situé à quatre-vingt kilomètres de son domicile). Il prétendait également que le "changement" qui interviendrait dans le soutien dont il bénéficiait jusqu'ici au Royaume-Uni emporterait le risque réel de l'exposer à un traitement dégradant s'il était renvoyé. Eu égard à I'affaire $\mathrm{D}$. contre Royaume-Uni, la Cour juge que ces éléments ne sont pas déterminants dans l'appréciation d'un risque réel d'être soumis à un traitement inhumain et dégradant, dès lors qu'en l'espèce M. Bensaid avait de la famille en Algérie (à la différence de M. D. dans I'affaire précitée). Elle juge ces arguments spéculatifs, tout comme elle le fera dans I'affaire N. contre Royaume-Uni. L'affaire étant relative aux soins de santé mentale (et non à une maladie davantage susceptible de provoquer le décès, tel que le VIH au début des années 2000), il semble toutefois que la CEDH assouplit quelque peu sa jurisprudence : elle considéra en effet que "l'aggravation» extrême de l'état de santé mentale de M. Bensaid et les souffrances l'accompagnant pouvaient "en principe" être considérés comme un traitement inhumain 
ou dégradant s'il était renvoyé dans son pays d'origine, sans référence à une mort certaine. Dans I'affaire Aswat contre Royaume-Uni, M. Aswat, détenu au Royaume-Uni pour des actes de terrorisme et souffrant d'une schizophrénie paranoïaque, soutenait devant la $\mathrm{CEDH}$ que son extradition imminente vers les États-Unis pour y être jugé et détenu dans une prison de très haute sécurité, constituerait un traitement inhumain et dégradant risquant d'aggraver sa schizophrénie. Selon la Cour, à la lumière des preuves médicales actuelles, il existait un risque réel que l'extradition du requérant vers un autre pays et vers un environnement carcéral différent, potentiellement plus hostile, se traduise par une détérioration significative de son intégrité mentale et physique en mesure d'atteindre le seuil de l'article 3. La Cour exclut alors l'état de santé critique de son appréciation générale, ce qui s'explique par la nature même de l'extradition, entraînant un déracinement plus fort que le retour d'une personne dans son pays d'origine.

Si les conditions de détention ne sont pas compatibles avec l'état de santé mentale de l'étranger, notamment eu égard à la durée d'incarcération, l'État partie peut être condamné pour violation de l'article 5 paragraphe 1 de la Convention relatif au droit à la liberté et à la sûreté, ce qui ne fut pas retenu dans I'affaire Thimothawes contre Belgique (2017). Demandeur d'asile débouté en état dépressif abandonnique et atteint de divers troubles psychologiques, $M$. Thimothawes forma un recours contre la Belgique devant la CEDH après avoir été placé dans un centre de transit à la frontière belge dans l'attente de son expulsion vers la Turquie (pays de provenance), à la suite d'un refus d'entrée. Cette rétention administrative n'était pas, selon lui, compatible avec son état de santé mental. Selon la Cour, il avait pourtant bien bénéficié d'une "attention particulière" dans les deux centres de transit où il était retenu, ce qui excluait toute violation de l'art. 5 paragraphe 1, f) de la Convention (détention d'un étranger non admis à l'entrée sur le territoire).

Dans des affaires excluant l'extradition ou la question de la santé mentale, la Cour confirma son appréciation restrictive. La troisième affaire ( $\mathrm{N}$. c. Royaume-Uni) concernait une ressortissante ougandaise, Mme N., également infectée par le VIH et souffrant de lourdes pathologies. Déboutée de l'asile au Royaume-Uni, elle risquait un retour forcé vers son pays d'origine. La CEDH a toutefois considéré que la situation de Mme N. n'était pas suffisamment exceptionnelle pour atteindre le seuil de gravité exigé et ainsi empêcher son renvoi. À la différence de M. D., elle ne présentait pas un risque réel de mourir : son état de santé, stable grâce au traitement antirétroviral dont elle bénéficiait au Royaume-Uni, ne revêtait aucun caractère critique, et elle était apte à voyager. Dès lors, l'éloignement, s'il était réalisé, n'emporterait pas violation par le Royaume-Uni de l'article 3 de la Convention. En 2012, la CEDH est parvenue à la même conclusion dans un arrêt faisant suite à une plainte contre la Belgique de Mme Yoh-Ekale Mwanje, atteinte du virus VIH depuis des années et à un stade avancé de l'affection.

Onze ans après I'affaire D. contre Royaume-Uni, I'affaire N. contre Royaume-Uni est l'occasion pour la Cour d'apporter pour la première fois une définition - négative - de la notion de circonstances très exceptionnelles : ne présentent pas selon elle un tel caractère les circonstances faisant état de l'existence d'un traitement médical dans le pays d'origine : Mme $N$. bénéficiait d'un traitement en Ouganda, bien qu'il était difficile d'établir la mesure dans laquelle 
elle pourrait l'obtenir et s'il était réellement approprié à son état de santé (tout comme M. Bensaid). En outre, le fait que l'éloignement vers l'Ouganda réduirait significativement l'espérance de vie de Mme $\mathrm{N}$. et dégraderait de manière importante son état de santé s'il était rendu effectif ne revêt pas non plus selon la Cour un caractère " très exceptionnel " justifiant la suspension du renvoi.

Le risque réel de mauvais traitements, pour être actuel ou imminent pour sa santé s'il était éloigné, suppose à l'époque pour la Cour l'absence totale de soutien dans le pays d'origine et un état de santé extrêmement critique qui emporterait de manière certaine le décès du requérant. D'ailleurs, dans l'affaire D. contre Royaume-Uni, la CEDH s'était davantage fondée sur le risque réel pour M. D de mourir que sur la rapidité avec laquelle son état de santé se dégraderait en cas de renvoi ou l'absence de soutien dans le pays de destination (qui constituaient toutefois des éléments d'appréciation importants). Dans l'affaire N. c. Royaume-Uni, la Cour se fonda également sur le motif socio-économique tel que soutenu par le Royaume-Uni : en dépit de sa nature absolue ne supportant en principe aucune dérogation, l'interdiction des traitements inhumains ou dégradants ne peut faire peser sur les États une charge trop lourde en leur faisant obligation d'offrir des soins de santé à tous les étrangers en situation irrégulière sur leur territoire. De ce point de vue, les États n'ont pas l'obligation de pallier les disparités entre leur système de soins, plus favorable, et le niveau de traitement existant dans le pays où le migrant malade risque d'être éloigné (JulienLaferrière, 2009). C'est pourquoi I'OMS préconise depuis longtemps de résoudre les défis sanitaires posés par les mouvements de population, en particulier pour les systèmes de santé des États. En effet, si les restrictions aux droits humains dans le domaine de la santé peuvent constituer des facteurs d'émigration (une personne malade qui ne peut bénéficier des soins adéquats dans son pays d'origine sera poussée à émigrer en dehors de celui-ci), les migrations peuvent à l'inverse conduire à de telles restrictions pour les réfugiés, les demandeurs $d^{\prime}$ asile et de manière générale tous les migrants qui s'y livrent. II revient aux États d'origine, de transit et de destination de faire en sorte d'améliorer l'accès à leur système de santé (OMS, 2017).

Dans I'affaire S. J. contre Belgique, Mme S. J, ressortissante nigériane en situation irrégulière en Belgique et atteinte du $\mathrm{VIH}$, risquait un éloignement du territoire belge accompagnée de ses trois enfants après s'être vue refuser sa demande de régularisation. Elle alléguait d'un risque réel $d^{\prime}$ 'être soumis à un traitement contraire à l'article 3 , ce que la Cour réfuta: Mme S. J. ne se trouvait pas dans un état critique, était apte à voyager et l'évolution de sa maladie était alors sous contrôle grâce à l'administration de médicaments en Belgique. Elle conclut que, si le cas de Mme S. J. était marqué par de fortes considérations humanitaires militant en faveur d'une régularisation de son séjour, ces considérations n'étaient "pas tellement impérieuses" qu'elles s'opposeraient, sous l'angle de l'article 3 de la Convention, à son retour dans son pays d'origine. L'examen de l'appréciation de la Cour, en particulier au regard de l'affaire Aswat, peut alors paraître difficile à justifier : la juge Power-Forde, dans son opinion dissidente, souligna à ce titre qu' "à I'instar de I'affaire Aswat, [Mme S. J.] se retrouvera certainement dans un "environnement plus hostile" qui affectera sa santé ". Dès lors, "il n'est pas logique que la même Cour dise que [l'article 3] n'interdit pas l'expulsion d'une mère vulnérable vers un État tiers où les conditions lui seront fatales". 
Eu égard à toutes ces constatations, la Cour distinguait clairement l'hypothèse d'un éloignement dans le cadre d'une mort imminente, de l'éloignement faisant face à une mort proche ou quasi certaine, ce qui souleva à l'époque des débats au sein même de la Grande chambre chargée de juger l'affaire N. contre Royaume-Uni ${ }^{6}$, et longtemps après ${ }^{7}$. Une telle approche ne permettait que très rarement aux migrants gravement malades de se maintenir temporairement sur le territoire, même atteints d'une maladie incurable. Pis, l'appréciation de la Cour risquait de porter gravement atteinte à l'effectivité de l'interdiction des traitements humains ou dégradants. En témoigne le décès de Mme $\mathrm{N}$. peu de temps après son arrivée en Ouganda, que la CEDH avait laissée expulser par le Royaume-Uni. C'est à I'occasion de l'examen du recours d'un étranger en situation irrégulière atteint d'un cancer que la Grande chambre de la CEDH assouplit sa position en 2016, dans I'affaire Paposhvili contre la Belgique ${ }^{8}$ (Klausser, 2017).

\section{Une position jurisprudentielle plus favorable au migrant malade exposé à l'éloignement}

À la suite de la notification d'un ordre de quitter le territoire belge après deux refus de l'office des étrangers de régulariser sa situation administrative pour motifs médicaux, M. Paposhvili risquait un retour forcé vers la Géorgie, son pays d'origine. Atteint d'une maladie très grave, une leucémie lymphoïde chronique engageant son pronostic vital, il forma une demande de suspension de l'éloignement et d'annulation des refus de régularisation devant le Conseil belge du contentieux des étrangers. Selon lui, son renvoi dégraderait davantage son état de santé déjà préoccupant. Dans l'attente de la décision du Conseil, il forma une demande de mesure provisoire auprès de la CEDH visant à suspendre en urgence son éloignement, au motif que son renvoi emporterait violation de l'article 3 de la Convention. La CEDH ordonna au gouvernement belge de suspendre la mesure de renvoi le temps de la procédure nationale. Finalement, le Conseil rejeta sa demande. Décédé entre temps, sa famille poursuivit sa requête devant la $\mathrm{CEDH}$, qui accepta d'examiner la requête sur le fond en dépit du décès de $\mathrm{M}$. Paposhvili. Elle affirme que les droits et libertés consacrés dans la Convention ne peuvent se muer en des garanties "théoriques et illusoires" du fait d'une interprétation et d'une application trop strictes de la Convention. À cet égard, la juridiction finit par définir puis assouplir le champ d'application des cas très exceptionnels mentionnés dans l'arrêt N. contre Royaume-Uni. Pour contrer les arguments des proches de M. Paposhvili, le gouvernement belge se fonde sur la jurisprudence antérieure de la Cour, c'est-à-dire l'appréciation d'un seuil de gravité par référence à un état de santé critique au moment du renvoi. En outre, le gouvernement belge alléguait qu'il existait des garanties suffisantes au bon traitement de l'individu en Géorgie, dès lors que les autorités locales

\footnotetext{
$6 \mathrm{CEDH}$, N. c. Royaume-Uni, opinion dissidente commune aux juges Tulkens, Bonello et Spielmann, para. 23

7 Dans l'affaire similaire Yoh-Ekale Mwanje (voir supra), certains juges appelaient la Grande Chambre de la Cour à réexaminer la jurisprudence N. contre Royaume-Uni dans leur opinion séparée partiellement concordante : "la différence entre une personne qui est sur son lit de mort ou dont on sait qu'elle est condamnée à bref délai nous paraît infime en termes d'humanité. Nous espérons que la Cour puisse un jour revoir sa jurisprudence sur ce point".

$8 \mathrm{CEDH}$, Paposhvili c. Belgique.
} 
auraient été informées des particularités de son état de santé ou auraient reçu la liste des traitements médicaux requis si le renvoi avait effectivement été mis en œuvre.

La Cour rappelle les principes généraux applicables à l'éloignement d'un étranger malade : pour se conformer à l'obligation négative que revêt l'article 3 et déterminer si M. Paposhvili risque d'être exposé à un traitement inhumain ou dégradant en cas de renvoi, la Belgique doit se conformer à l'obligation positive consistant à apprécier les conséquences "prévisibles " d'un tel renvoi sur l'individu. Cela nécessite alors de comparer la situation qui est celle de M. Paposhvili avant le renvoi, et qui serait la sienne après le renvoi. Plusieurs facteurs doivent alors être pris en compte, au cas par cas. D'une part, la situation générale dans le pays de destination, en particulier si les soins généralement disponibles y sont suffisants et adéquats en pratique pour traiter la pathologie dont souffre l'intéressé afin d'éviter qu'il soit exposé à un traitement contraire à l'article 3. $D^{\prime}$ 'autre part, l'analyse des circonstances propres à la situation du requérant, sans qu'il ne se trouve nécessairement dans un état critique. Les autorités belges et les instances nationales compétentes n'ont examiné les données médicales de M. Paposhvili et l'impact de l'éloignement sur son état de santé dans aucune des procédures diligentées. Or, tant en ce qui concerne la situation générale en Géorgie que la situation de M. Paposhvili, la CEDH relève d'une part l'absence, dans le pays de destination, d'un traitement adéquat (le seul traitement curatif pour cette maladie, en l'occurrence une greffe allogénique, n'était pas disponible dans le pays d'origine) et d'autre part son inaccessibilité (M. Paposhvili n'aurait pas pu bénéficier d'autres traitements en raison du prix, de la localisation géographique, des difficultés de mobilité dues à son état de santé, et de l'absence de soutien familial dans ce pays). Ce, alors même qu'un certificat du médecin traitant de l'intéressé attestait que son cancer serait "potentiellement mortel à court terme" en cas d'arrêt du traitement. Du reste, l'épouse du requérant et ses enfants se trouvaient tous en Belgique, M. Paposhvili ne disposant d'aucune famille en Géorgie. Surtout, et c'est d'ailleurs ce qui modifie fondamentalement la position de la Cour, celle-ci accepte de retenir comme circonstances très exceptionnelles le fait que M. Paposhvili produise des motifs sérieux de croire qu'il aurait été exposé à un " déclin grave, rapide et irréversible " de son état de santé, entraînant des "souffrances intenses " ou une "réduction significative de son espérance de vie ", si l'éloignement avait été rendu effectif. En tant que tel, le risque de décès certain et imminent ne fonde plus l'appréciation de la Cour : la Cour écarte donc l'argument du gouvernement belge relatif au fait que le requérant remplissait les conditions médicales pour voyager et ne se trouvait pas dans un état critique. À la différence des juges de la cinquième section de la Cour, qui conclurent respectivement à l'unanimité et à la majorité la non-violation des articles 3 et 8 de la Convention, la Grande chambre considéra qu'il y aurait eu violation de l'article 3 de la Convention par la Belgique si le requérant avait été éloigné vers la Géorgie sans évaluation de toutes ces données.

Faut-il en déduire un abandon de sa jurisprudence antérieure? Rien n'est moins sûr, eu égard au critère d'irréversibilité. D'autant qu'il convient de rappeler qu'encore une fois, conformément à sa compétence subsidiaire et à la souveraineté étatique, la CEDH n'aurait pas eu compétence pour imposer à la Belgique de régulariser la situation de $\mathrm{M}$. Paposhvili, seule compétente pour apprécier les 
conditions de séjour sur son territoire. II reste que sur le terrain des obligations négatives et positives découlant de l'article 3, la Cour aurait pu contraindre la Belgique à suspendre l'éloignement de $\mathrm{M}$. Paposhvili jusqu'à son décès.

Au niveau de I'UE, la CJUE a également eu l'occasion, plus rare, de se prononcer sur l'éloignement par un État membre d'un ressortissant de pays tiers gravement malade, dans le cadre des questions préjudicielles posées par les États membres. L'article 19 paragraphe 2 de la Charte protège expressément tout ressortissant de pays tiers contre un éloignement, une expulsion ou une extradition à partir du territoire de I'UE, vers un État où il existe un risque sérieux qu'il soit soumis à la peine de mort, à la torture ou à d'autres peines ou traitements inhumains ou dégradants. Par le biais de la question préjudicielle posée par la Cour du travail de Bruxelles dans I'affaire Abdida, elle conclut que l'éloignement de M. Abdida, gravement malade, devrait impérativement être suspendu par la Belgique si son exécution était susceptible d'exposer le ressortissant de pays tiers à un risque sérieux de détérioration grave et irréversible de son état de santé ${ }^{9}$, deux ans avant I'affaire Paposhvili devant la Grande chambre de la $\mathrm{CEDH}$. Elle conclut qu'à défaut, l'éloignement serait susceptible de constituer un traitement inhumain ou dégradant au sens de l'article 19 paragraphe 2 et de l'article 4 de la Charte.

La suspension de l'éloignement du migrant en situation irrégulière gravement malade peut également être prononcée sur le fondement de droits dits "relatifs", consacrés dans la Convention et la Charte. Si aucun seuil de gravité n'est ici exigé, leur protection est plus difficile à envisager, la Convention autorisant expressément des ingérences à ces droits de la part des États.

\section{L'éloignement du migrant malade sous l'angle du respect de la vie privée et familiale et du droit à un recours effectif}

Dans certaines circonstances, l'éloignement d'un migrant malade peut soulever une question sous l'angle du droit à la vie privée et familiale lorsqu'il est susceptible de constituer une atteinte suffisamment grave à son intégrité physique ou morale ou au libre exercice de la vie familiale. Sous l'angle procédural, l'éloignement d'un migrant malade peut également constituer une atteinte au droit à un recours effectif, également qualifié de droit " relatif ».

\section{La prise en compte de la vie privée et du maintien de la vie familiale du migrant malade au moment de son éloignement (article 8 de la Convention et article 7 de la Charte)}

À la différence de l'article 3, le droit au respect de la vie privée et familiale est soumis à des dérogations : I'article 8-2 de la Convention autorise certaines ingérences de l'autorité publique dans l'exercice de ce droit, lorsqu'elles sont proportionnées et nécessaires à certains impératifs. Une telle ingérence doit être prévue par la loi et constituer une mesure nécessaire à la sécurité nationale, à la sûreté publique, au bien-être économique du pays, à la défense de l'ordre et à

9 CJUE, Centre public d'action sociale d'Ottignies-Louvain-la-Neuve c. Moussa Abdida, point 53. 
la prévention des infractions pénales, à la protection de la santé ou de la morale ou à la protection des droits et libertés d'autrui dans une société démocratique. Dans l'exercice de sa compétence subsidiaire, la CEDH s'assure toutefois que l'État partie à la Convention a ménagé un juste équilibre entre les intérêts en présence : ceux du migrant, et ceux de l'État. II s'agit d'abord pour la CEDH d'étudier si les circonstances, telles qu'une décision d'éloignement, entrent dans le champ d'application des dérogations autorisées par la Convention (sécurité nationale, prévention des infractions pénales, etc.). Elle évaluera ensuite les conséquences potentielles de l'éloignement sur l'individu gravement malade en se référant à l'atteinte portée à son intégrité physique ou morale (vie privée) ou à l'unité de la vie familiale (vie familiale).

En ce qui concerne l'atteinte à l'intégrité physique et morale, celle-ci doit être suffisante pour relever de l'article 8 , sans pour autant constituer un acte de torture ou de mauvais traitements. À la différence de ses décisions relatives à I'article 3, la CEDH ne se réfère pas uniquement aux conséquences irréversibles du renvoi sur l'état de santé du migrant. La CEDH n'exclut pas que l'éloignement puisse nuire, à un degré suffisant, à l'intégrité physique et/ou morale du migrant malade au point d'enfreindre l'article 8 , alors même qu'il ne présente pas une " gravité " telle qu'il emporterait violation de I'article 3. Ainsi, dans I'affaire Bensaid, la CEDH consent à évaluer les conséquences de l'expulsion en Algérie de M. Bensaid sur son intégrité morale, sous l'angle de l'article 8. Celui-ci alléguait notamment que, privé des soins habituellement reçus au Royaume-Uni, son état de santé mental (schizophrénie) risquerait d'empirer s'il retournait en Algérie. La Cour rejette son argumentaire : I'ingérence des autorités britanniques dans sa vie privée (imposer de quitter un territoire où l'intéressé, malade, a vécu onze années consécutives et où il a noué des relations personnelles) est une mesure proportionnée au but poursuivi (notamment le bien-être économique du pays) et nécessaire dans une société démocratique. Le bien-être économique du pays est une dérogation expressément prévue par la Convention au droit au respect de la vie privée, régulièrement opposée par les États pour justifier l'éloignement d'un migrant malade. Cela signifie que lorsque le maintien sur le territoire d'un migrant malade en situation irrégulière fait peser une charge trop importante sur l'État, la CEDH peut écarter la violation de l'article 8, sauf si l'éloignement risque d'emporter des conséquences irréversibles sur son intégrité physique ou morale. Ainsi, la CEDH n'exclut pas que l'éloignement puisse constituer un bouleversement dans la vie du requérant pouvant porter, en soi, atteinte à sa vie privé $e^{10}$. Elle reconnaît expressément que le droit au respect de la vie privée suppose de protéger l'épanouissement personnel de l'individu vis-à-vis du monde qui l'entoure (y compris sur un territoire étranger), et que la protection de la stabilité mentale constitue bien un préalable inéluctable à celui-ci. Encore une fois, tout dépendra des circonstances toutes particulières de l'espèce, l'étendue des obligations étatiques pouvant varier en fonction de chacune d'elles. Ainsi, en ce qui concerne l'extradition d'un étranger malade vers un pays étranger, les circonstances paraissent différentes pour la CEDH : dans I'affaire Aswat contre Royaume-Uni, elle considère que l'extradition conduira à ce que $\mathrm{M}$. Aswat soit transféré vers un pays où il n'a aucun lien, où il sera détenu et où il n'aura pas

$10 \mathrm{CEDH}$, Bensaid c. Royaume-Uni, para. 48. 
le soutien de sa famille et de ses amis ${ }^{11}$, soit dans un environnement potentiellement plus hostile, à la différence d'une simple mesure de renvoi vers le pays d'origine.

En ce qui concerne le droit au respect de la vie familiale, la CEDH évalue si le renvoi constitue une entrave à la jouissance ou à l'établissement de la vie familiale dans le pays d'origine (elle apprécie l'existence $d^{\prime}$ ' obstacles insurmontables " ${ }^{12}$ à ce que la famille du migrant malade le rejoigne et vive avec lui dans le pays d'origine), l'étendue des liens que les personnes concernées ont avec l'État partie en cause, et si la mesure répond au contrôle de l'immigration ou à des considérations d'ordre public. Pris isolément, ces facteurs pourront toutefois difficilement permettre de caractériser une violation de I'obligation positive pour l'État de maintenir l'unité familiale sur son territoire. Ainsi, l'absence de soutien familial dans le pays d'origine pour une femme atteinte d'une maladie mentale ne constitue pas un obstacle insurmontable au renvoi : I'ingérence des autorités allemandes dans la vie privée et familiale de Mme Khan, fondée sur la sauvegarde de la sûreté publique, était proportionnée et nécessaire (Khan contre Allemagne, 2017). Dans I'affaire S. J. contre Belgique, en sus d'une violation de I'article 3, Mme S. J alléguait que la Belgique était le seul pays où elle pourrait exercer une vie familiale normale et conforme à l'article 8 de la Convention avec son partenaire et leurs enfants (nés en Belgique). Elle considère que I'unité familiale faisait obstacle à son renvoi et à celui de ses trois jeunes enfants dès lors que ces derniers risqueraient de se retrouver livrés à eux-mêmes avec une mère malade, puis orphelins, dans un pays où ils ne bénéficient pas de réseau social ni familial. La Cour estime que le grief tiré d'une violation de l'article 8 n'est pas fondé. Elle constate que la relation existante entre cette mère et ses enfants constitue bien une vie familiale au sens de l'article 8 (faisant parfois peser sur l'État d'accueil l'obligation de développer et de maintenir cette vie familiale, par exemple en réexaminant une demande de régularisation). Toutefois, elle considère que la capacité d'adaptation des enfants était encore suffisamment grande pour qu'une réinstallation dans le pays d'origine de leurs parents soit réaliste. La Cour balaie également l'argument de la requérante relatif à l'incapacité pour cette mère de s'occuper de ses enfants en bas-âge, du fait qu'elle n'invoque aucun élément qui démonterait que le père de famille, en séjour irrégulier en Belgique, ne pourrait pas accompagner Mme S. J. et leurs enfants au Nigéria. Sur ce dernier point, la Cour opère un raisonnement somme toute hypothétique, qu'elle reproche précisément aux requérants lorsqu'elle apprécie l'existence d'un risque réel et grave de subir un traitement inhumain ou dégradant en cas de renvoi (article 3, Bensaid contre Royaume-Uni, supra). Toutefois, il est vrai qu'en l'espèce rien ne militait contre l'existence d'une vie familiale effective au Nigéria. Surtout, à aucun moment un État partie ne doit avoir pour obligation de régulariser une mère de famille du seul fait d'un risque potentiel de décès et/ou des difficultés à maintenir l'unité familiale dans le pays où elle est susceptible d'être envoyée : cette question dépasse largement le champ de protection couvert par la Convention et relève d'une appréciation discrétionnaire des États parties (régularisation exceptionnelle pour motifs

$11 \mathrm{CEDH}$, Aswat c. Royaume-Uni, para. 57.

$12 \mathrm{CEDH}$, S.J. c. Belgique. 
familiaux par exemple ${ }^{13}$ ). Elle écarte toute ingérence arbitraire des autorités belges dans le droit au respect de la vie familiale.

L'opinion dissidente de la juge Power-Forde dans cette affaire soulève toute la difficulté d'apprécier l'étendue des obligations positives et négatives découlant du droit au respect de la vie familiale : "[I]ndépendamment des horribles souffrances physiques qu'entraîne un décès dû au sida, cette requérante devra en plus subir de profondes souffrances émotionnelles lorsqu'elle regardera ses trois jeunes enfants assister à la détérioration de l'état et au décès de leur mère à cause d'un manque de médicaments, tout en sachant qu'elle sera incapable de les aider et qu'elle les laissera orphelins après son décès". Elle souligne que la Belgique avait I'intention délibérée de placer la requérante dans une telle situation, dès lors que "[I]e fait crucial qui déterminera si elle vivra ou mourra est l'exécution de la décision d'expulsion prise par l'État", ce qui aurait dû conduire la Cour à admettre la violation de l'article 3 et 8 . Elle appelait alors la Grande Chambre à mettre un terme à ces divergences d'interprétation, ce qu'elle tentera de faire en 2016 dans I'affaire Paposhvili contre Belgique, sous I'angle de I'article 8 de la Convention (voir supra en ce qui concerne l'article 3). En l'espèce, le renvoi du requérant vers la Géorgie aurait emporté selon ses proches une atteinte au droit au respect de sa vie familiale. La Cour a considéré que la question centrale était de savoir si les autorités belges compétentes avaient l'obligation d'autoriser le requérant à séjourner en Belgique et de demeurer auprès de sa famille pour le temps qui lui restait à vivre. Cette question était toutefois nécessairement liée à une autre : le renvoi de M. Paposhvili en Géorgie aurait-il emporté violation du droit au respect de sa vie familiale s'il avait été mis en œuvre? En somme, la Belgique avait l'obligation d'évaluer deux choses : si la situation du requérant permettait de considérer qu'on pouvait raisonnablement s'attendre à ce que sa famille le suive en Géorgie d'une part, et si, à défaut, le droit au respect de la vie familiale consacré par la Convention exigeait que la Belgique autorise son séjour provisoire jusqu'à son décès (imminent) d'autre part. La Cour tranche rapidement ces questions : elle souligne que l'article 8 comportait une obligation positive procédurale pour les autorités belges d'évaluer la situation concrète de M. Paposhvili au moment du renvoi, en particulier l'impact de l'éloignement sur sa vie familiale, compte tenu du degré de dépendance du requérant envers sa famille en raison de la dégradation de son état de santé en Belgique. Le requérant est décédé avant que la Belgique ne procède à son renvoi, mais la Cour constate que si la Belgique avait poursuivi l'éloignement du requérant vers la Géorgie sans évaluation de ces données, il y aurait eu violation de l'article 8 de la Convention.

\section{La protection procédurale du migrant malade exposé à l'éloignement (article 13 de la Convention et article 47 de la Charte)}

Dans des affaires où il s'est agi pour la CEDH d'examiner la suspension de l'éloignement imminent d'un migrant malade et/ou d'apprécier l'étendue de la responsabilité de l'État partie après la mise en œuvre de l'éloignement, les requérants prétendaient régulièrement n'avoir pas bénéficié de garanties procédurales suffisantes au stade de la remise en cause de la décision admi-

13 Voir notamment l'article L 313-11 7 CESEDA, en droit français. 
nistrative d'éloignement, alléguant d'une violation de l'article 13 (droit à un recours effectif), généralement combiné avec la violation de l'article 3 ou 8 de la Convention. À la différence du droit à un procès équitable, le droit à un recours effectif bénéficie en principe aux étrangers en situation irrégulière sur le territoire d'un État partie. De manière générale, un recours sera considéré comme effectif sur la scène nationale s'il est accessible, disponible et qu'il permet un examen rigoureux et qualitatif du contenu du grief allégué. Le migrant doit être en mesure de voir les motifs impérieux militant contre son renvoi susceptible de porter atteinte à ses droits (de mener une vie familiale normale, de ne pas subir de mauvais traitements, etc.) être examinés par un juge indépendant et impartial.

Le recours n'a pas nécessairement besoin d'être assorti d'un effet suspensif pour être effectif. Toutefois, la CEDH a déjà sanctionné la Belgique sur ce point dans I'affaire précitée S. J. contre Belgique. Mme S. J. alléguait d'un risque réel de subir un traitement inhumain ou dégradant en raison de sa situation médicale si elle était renvoyée au Nigéria, alors que le recours offert par le droit belge contre la mesure d'éloignement forcée n'était assorti d'aucun effet suspensif. Dès lors, elle avait été éloignée du territoire avant de voir son grief examiné. À défaut de constater la violation de l'article 3 pris isolément (voir supra), les juges conclurent à l'unanimité qu'en raison du caractère irréversible de l'éloignement et de l'importance du droit protégé par l'article 3, l'éloignement avait emporté violation de l'article 13 combiné à l'article $3^{14}$.

En ce qui concerne le droit de I'UE, la CJUE interpréta la Directive Retour comme imposant aux États membres de conférer un effet suspensif aux recours formés par un ressortissant d'un pays en séjour irrégulier contre une décision de retour, dans certaines circonstances liées à l'état de santé. Dans I'affaire Abdida (voir supra), la CJUE souligna que l'exécution du renvoi de M. Abdida vers le Niger, ressortissant nigérien souffrant d'une grave maladie, était susceptible de l'exposer à un risque sérieux de détérioration grave et irréversible de son état de santé. Or, le recours belge n'étant assorti d'aucun effet suspensif, la CJUE conclut que s'il devait être mis en œuvre, l'éloignement constituerait un traitement inhumain ou dégradant au sens de l'article 19-2 de la Charte, combiné à I'article $47^{15}$. Cette décision est importante, dès lors que la Directive Retour laisse en principe le choix discrétionnaire aux États membres de I'UE de conférer ou non un effet suspensif aux recours formés par un ressortissant de pays tiers en situation irrégulière contre une décision visant à l'éloigner de leur territoire.

\section{Conclusion}

Depuis les années 80, la jurisprudence de la CEDH, suivie de la CJUE, a contribué à consacrer un socle de droits minimums aux migrants en situation irrégulière gravement malades sur le territoire européen. Sans parvenir à parachever cette construction, les États membres de l'ONU (dont la totalité des États européens), se sont accordés, en adoptant le Pacte mondial pour les migrations

14 CEDH, S.J. c. Belgique, para. 106.

15 CJUE, Centre public d'action sociale d'Ottignies-Louvain-la-Neuve c. Moussa Abdida, point 64 . 
en décembre $2018^{16}$, à promouvoir davantage la santé physique et mentale des migrants dans leurs politiques nationales ${ }^{17}$.

La jurisprudence européenne ne constitue pas un bouclier de protection suffisant contre toutes les formes d'atteintes ultérieures aux droits des migrants malades à la frontière ou sur le territoire européen. Aucune de ces deux juridictions ne peut imposer à un État partie à la Convention ou à I'UE d'admettre à l'entrée ou de régulariser la situation d'un ressortissant de pays tiers en situation irrégulière pour un motif médical. En droit de I'UE, une telle régularisation pour "des motifs charitables, humanitaires ou autres" (Directive Retour) est discrétionnaire. D'ailleurs, la Charte ne peut conférer un droit automatique au migrant malade dont l'éloignement a été reporté à bénéficier de la protection subsidiaire de I'UE pour ce seul motif ${ }^{18}$ (cette protection issue du droit de I'UE est en principe accordée à certains étrangers vulnérables alléguant d'un risque de torture ou de mauvais traitements dans leur pays d'origine). Cela peut placer le migrant malade dans un "twilight status" (Martin, 2005), parallèle aux statuts juridiques officiellement admis : toléré à la frontière ou sur le territoire aussi longtemps que le motif justifié de non-retour perdure (soit dans certains cas des années), il ne bénéficie d'aucun statut légalisant sa présence, ce qui le prive du bénéfice de certaines aides sociales.

À défaut pour le droit européen des droits de l'Homme de consacrer une position claire à la hauteur de ses ambitions, les États européens disposent encore d'un large pouvoir d'appréciation relatif au droit à la protection de la santé du migrant en situation irrégulière. À cet égard, la jurisprudence européenne freine difficilement l'élan collectif de certains États européens relatif au durcissement de la lutte contre l'immigration irrégulière (contrôles accrus en ce qui concerne les demandes de régularisation pour motifs médicaux; contrôles d'immigration au sein même des hôpitaux, en particulier en Allemagne). La France n'y fait évidemment pas obstacle : en transférant la compétence de l'évaluation médicale des demandeurs de titres de séjour pour motifs médicaux des ARS (ministère de la Santé) à un collège de médecins du service médical de l'OFII (ministère de I'Intérieur), la réforme de mars 2016 continue de faire l'objet de violentes critiques de la part d'ONG et d'associations de défense des droits des personnes étrangères en situation irrégulière. Le législateur semble avoir tenu compte de ces considérations : la loi $n^{\circ} 2018-778$ du 10 septembre $2018^{19}$ prévoit désormais que les médecins compétents ne peuvent demander les informations médicales nécessaires aux professionnels de santé concernés que sous réserve de l'accord de l'étranger, et dans le respect des règles de déontologie médicale. Du reste, si l'avis favorable du collège de médecins de l'OFIl en ce qui concerne la régularisation de l'individu ne lie toujours pas le préfet, la loi impose désormais à ce dernier de motiver de manière spéciale toute décision de refus en présence d'un tel avis. Ces dispositions sont entrées en vigueur le $1^{\text {er }}$ mars 2019.

16 Tout comme la Déclaration de New York pour les réfugiés et les migrants, ce texte est non contraignant. S'il illustre la construction progressive d'un Droit des migrations, la question des droits individuels demeure toutefois accessoire à celle de la gestion du phénomène migratoire au sens large.

17 Pacte mondial pour des migrations sûres, ordonnées et régulières, objectif 15, para. 31, e). 18 CJUE, M'Bodj c. État belge, point 41.

19 Loi n $2018-778$ du 10 septembre 2018 pour une immigration maîtrisée, un droit d'asile effectif et une intégration réussie, art. 56. 


\section{Références bibliographiques}

\section{Principaux arrêts et jugements cités}

CJUE

Centre public d'action sociale d'Ottignies-Louvain-la-Neuve c. Moussa Abdida, 18 décembre 2014, aff. C-562/13.

Mohamed M’Bodj c. État belge, 18 décembre 2014, aff. C-542/13.

CEDH

Thimothawes c. Belgique, 4 avril 2017, req. $n^{\circ} 39061 / 11$.

Paposhvili c. Belgique, 13 décembre 2016, req. $n^{\circ}$ 41738/10.

Khan c. Allemagne, 23 avril 2015, req. n 38030/12.

Paposhvili c. Belgique, 17 avril 2014, req. $\mathrm{n}^{\circ}$ 41738/10.

S.J. c. Belgique, 27 février 2014, req. $\mathrm{n}^{\circ}$ 70055/10.

Aswat c. Royaume-Uni, 16 avril 2013, req. n 17299/12.

De Souza Ribeiro c. France, 13 décembre 2012, req. n²2689/07.

Yoh-Ekale Mwanje c. Belgique, 20 décembre 2011, req. $\mathrm{n}^{\circ}$ 10486/10.

N. c. Royaume-Uni, 27 mai 2008, req. n²6565/05.

Bensaid c. Royaume-Uni, 6 février 2001, req. n 44599/98.

D. c. Royaume-Uni, 2 mai 1997, req. n³0240/96.

\section{Pactes/rapports/recommandations}

Organisation des Nations Unies - Conférence intergouvernementale chargée d'adopter le Pacte mondial pour des migrations (2018) Pacte mondial pour des migrations sûres, ordonnées et régulières, A/CONF.231/3, Annexe.

OMS (2017) Promotion de la santé des réfugiés et des migrants, Rapport du Secrétariat, A70/24.

Agence des droits fondamentaux de I'Union européenne (2016) Asile et migration vers I'UE en 2015, Luxembourg, Office des publications de I'Union européenne, $40 \mathrm{p}$.

Agence des droits fondamentaux de l'Union européenne (2012) L'accès aux soins de santé des migrants en situation irrégulière dans 10 États membres de I'Union européenne, Luxembourg, Office des publications de I'UE, 72 p.

Agence des droits fondamentaux de l'Union européenne (2012) Rapport comparatif sur les droits fondamentaux des étrangers en situation irrégulière dans I'UE, Luxembourg, Office des publications de I'UE, $117 \mathrm{p}$.

Comité des Ministres du Conseil de l'Europe (2011) Recommandation CM/ Rec(2011)13 aux États membres sur la mobilité, les migrations et l'accès aux soins de santé. 
Assemblée Parlementaire du Conseil de l'Europe (2001) Recommandation 1503 (2001) sur les conditions sanitaires des migrants et des réfugiés en Europe.

\section{Articles}

Cognet Marguerité, Hoyez Anne-Cécile et Poiret Christian (2012) Éditorial : Expériences de la santé en migration : entre inégalités et discriminations, Revue Européenne des Migrations Internationales, 28 (2), pp. 7-10.

Gabarro Céline (2018) L'accès aux soins des étrangers en situation irrégulière : une prise en charge au titre de la pauvreté et de la compassion, Migrations société, 30 (171), pp. 93-104.

Julien-Laferrière François (2009) L'éloignement des étrangers malades : faut-il préférer les réalités budgétaires aux préoccupations humanitaires?, Rev. trim. dr. h., 77, pp. 261 et s.

Klausser Nicolas (2019) L'étranger gravement malade : un statut fragmenté pour des garanties augmentées?, La Revue des droits de l'Homme, 15 [en ligne] consulté le 20/01/2019. URL : http://journals.openedition.org/revdh/5338

Klausser Nicolas (2017) Malades étrangers : la CEDH se réconcilie (presque) avec elle-même et l'Humanité, La Revue des droits de I'Homme, [en ligne] consulté le 20/01/2019. URL : http://journals.openedition.org/revdh/2965

Klausser Nicolas (2016) Rejet expéditif par la CEDH de la requête d'un étranger malade en voie d'expulsion : Une Convention à deux vitesses?, La Revue des droits de l'Homme, [en ligne] consulté le 20/01/2019. URL : http://journals.openedition.org/revdh/1788

Martin David (2005) Twilight Statuses: A Closer Examination of the Unauthorized Population, Migration Policy Institute (MPI), Policy Brief, 17 (2), pp. 2-4, [online] last checked on 13/09/2018. URL : https://www.migrationpolicy.org/research/ twilight-statuses-closer-examination-unauthorized-population

Martinent Éric (2012) La santé des migrants : un droit universel, Revue de droit sanitaire et sociale, 10 (3), pp. 133-159.

Musso Dimitrijevic Sandrine (2000) L'accès aux soins des étrangers en situation précaire, Hommes et société, 14 (1225), pp. 88-93.

Roman Diane (Dir.) (2012) Les droits sociaux, entre droits de l'Homme et politiques sociales. Quels titulaires pour quels droits?, Paris, LGDJ, 200 p.

Slama Serge et Parrot Karine (2014) Étrangers malades : I'attitude de Ponce Pilate de la Cour européenne des droits de l'Homme, Plein droit, 28 (101), pp. 27-30. 